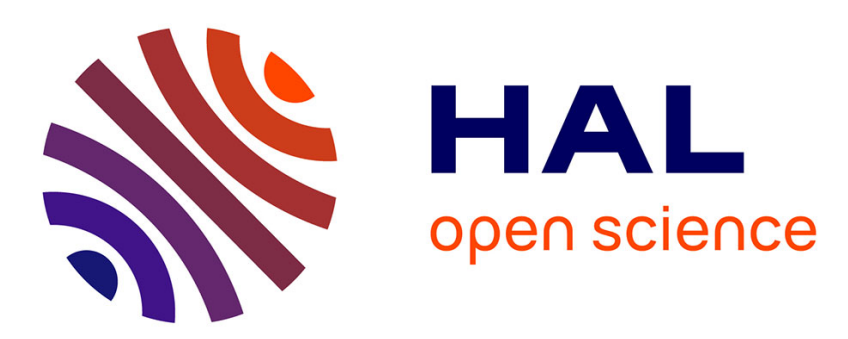

\title{
Composition chimique des bactéries libres ou adhérentes au contenu du rumen et du duodénum chez la vache
}

\author{
C Benchaar, C Bayourthe, M Vernay, R Moncoulon
}

\section{To cite this version:}

C Benchaar, C Bayourthe, M Vernay, R Moncoulon. Composition chimique des bactéries libres ou adhérentes au contenu du rumen et du duodénum chez la vache. Annales de zootechnie, 1995, 44 (Suppl1), pp.139-139. hal-00889301

\section{HAL Id: hal-00889301 https://hal.science/hal-00889301}

Submitted on 1 Jan 1995

HAL is a multi-disciplinary open access archive for the deposit and dissemination of scientific research documents, whether they are published or not. The documents may come from teaching and research institutions in France or abroad, or from public or private research centers.
L'archive ouverte pluridisciplinaire HAL, est destinée au dépôt et à la diffusion de documents scientifiques de niveau recherche, publiés ou non, émanant des établissements d'enseignement et de recherche français ou étrangers, des laboratoires publics ou privés. 


\title{
Composition chimique des bactéries libres ou adhérentes au contenu du rumen et du duodénum chez la vache
}

\author{
C Benchaar, C Bayourthe, M Vernay, R Moncoulon
}

\author{
ENSA, Laboratoire d'ingénierie agronomique, 31076 Toulouse, France
}

Chez le polygastrique, le flux bactérien duodénal est évalué par le biais d'un marqueur en prenant comme élément de référence la concentration de ce dernier dans un échantillon bactérien isolé à partir de la phase liquide (BAL) du rumen. Dans ce réservoir les BAL sont minoritaires par rapport aux bactéries fixées à la phase solide (BAS) et leur composition chimique diffère (Legay-Carmier et Bauchart, 1989, Br J Nutr, 61, 721-740). Peu d'informations concernent la microflore duodénale.

Quatre vaches Holstein en production munies de canules du rumen et du duodénum ont été utilisées au cours de 4 périodes expérimentales. Quotidiennement elles ont reçu, en 4 repas égaux, une ration à base d'ensilage de maïs $(56 \%)$, de foin de ray-grass $(11 \%)$ et de concentrés (43\%). L'isolement des BAL et la séparation des BAS ont été respectivement effectués selon les méthodes de Jouany et Thivend (1972, Ann Biol Biochim Biophys, 2, 673-677) et de Legay-Carmier et Bauchart (1989, Br J Nutr, 61, 721-740). La concentration en ARN a été déterminée par analyse calorimétrique des bases puriques, la teneur en azote $(\mathrm{N})$ par la technique de Kjeldhal. Les acides aminés (AA) ont été dosés par chromatographie après $24 \mathrm{~h}$ d'hydrolyse acide.

La composition chimique des fractions bactériennes isolées dans le rumen (BAL vs BAS) a été significativement différente ; ce qui n'est pas plus le cas dans le duodénum. Les teneurs en $\mathrm{MO}$ et $\mathrm{N}$ des BAL ruminales ont été inférieures à celles des BAS (-17 et -23\%) ; l'inverse a été observé pour l'ARN $(+35 \%)$. Par ailleurs, le taux de N-AA, ainsi que la proportion d'AA essentiels (E) et non essentiels (NE) ont été similaires. Le passage du rumen au duodénum a essentiellement modifié les teneurs en MO et ARN. II est possible que cette modification résulte de la contamination des BAL par des BAS. Pour ces dernières seule la concentration en ARN a varié $(+49 \%)$. Dans nos conditions expérimentales pour les $B A L$, quel que soit le site de prélèvement, et pour les BAS duodénales, le rapport ARN/Nt a été le plus élevé $(0,97)$. Une valeur inférieure a été enregistrée pour les BAS du contenu ruminal $(0,59)$. Or, dans ce réservoir les particules $\geq$ à $1 \mathrm{~mm}$ sont en proportion élevée, alors qu'elles ne le sont pas dans les contenus duodénaux. La teneur en ARN, exprimée en proportion de $\mathrm{Nt}$, serait un bon indice de l'activité de synthèse protéique de la cellule bactérienne (Bates et al, 1985, J Anim Sci, 61, 713-724). La différence de taille des particules-support pourrait s'accompagner d'une moindre activité métabolique des BAS ruminales. Yang (1991, thèse doctorat, Clermont-Ferrand) a également observé une augmentation de l'enrichissement ${ }_{15} \mathrm{~N}$ du BAS quand la taille des particules support diminue.

En conclusion, pour les différentes fractions bactériennes étudiées, les valeurs obtenues, notamment celles des rapports ARN/Nt, confirment l'intérêt du choix des BAL comme éléments de référence lors de l'estimation du flux duodénal bactérien.

\begin{tabular}{lccccc}
\hline & \multicolumn{2}{c}{ Rumen } & \multicolumn{2}{c}{ Duodénum } \\
\cline { 2 - 5 } Bactéries & BAL & BAS & BAL & BAS & ETR \\
\hline MO (\% MS) & 75,4 & 90,8 & 87,1 & 89,6 & 2,7 \\
N total (\% MS) & 6,1 & 7,3 & 6,8 & 6,4 & 0,5 \\
N-AA totaux $(\%$ Nt) & 82,5 & 84,7 & 80,2 & 81,9 & 3,2 \\
$\quad$ AAE $(\%$ AAt) & 50,9 & 50,5 & 50,7 & 51,3 & 1,5 \\
AANE (\% AAt) & 49,1 & 49,5 & 49,3 & 48,7 & 1,5 \\
ARN (\% MS) & 5,8 & 4,3 & 6,5 & 6,4 & 0,5
\end{tabular}

